\title{
Detection of Optic Disc Centre Point in Retinal Image
}

\author{
Latifah Listyalina $^{* 1}$, Dhimas Arief Dharmawan ${ }^{2}$ \\ ${ }^{1}$ Department of Electrical Engineering, Faculty of Science and Technology, Universitas Respati Yogyakarta \\ Jalan Laksda Adisucipto Km 6.3, Sleman 55281, Telp (0274) 488781 \\ ${ }^{2}$ Department of Electrical Engineering, Faculty of Engineering, Universitas Muhammadiyah Yogyakarta \\ Jalan Brawijaya, Geblangan, Tamantirto, Kasihan, Bantul 55183, Telp (0274) 387656
}

*Corresponding author, e-mail: listyalina@respati.ac.id

\begin{abstract}
Glaucoma and diabetic retinopathy $(D R)$ are the two most common retinal related diseases occurred in the world. Glaucoma can be diagnosed by measuring optic cup to disc ratio (CDR) defined as optic cup to optic disc vertical diameter ratio of retinal fundus image. A computer based optic disc is expected to assist the ophthalmologist to find their location which are necessary for glaucoma and DR diagnosis. However, many optic disc detection algorithms available now are commonly non-automatic and only work in healthy retinal image. Therefore, there is not information on how optic disc in retinal image of unhealthy patient can be extracted computationally. In this research work, the method for automated detection of optic disc on retinal colour fundus images has been developed to facilitate and assist ophthalmologists in the diagnosis of retinal related diseases. The results indicated that the proposed method can be implemented in computer aided diagnosis of glaucoma and diabetic retinopathy system development.
\end{abstract}

Keywords: Optic Disc, Retinal, Detection

\section{Introduction}

One of the modern medical imaging modalities is fundus photography. Fundus images are taken using fundus camera. Fundus images are useful to document the health of the optic disc, optic nerve, vitreous, fovea, macula, retina and eye's blood vessels [1]. Optic disc is one of the major parts of a retinal fundus image. Optic disc is the region in the retina which appears as a very bright region compared to its surrounding. In a circular area of optic disc, there are blood vessels and optic nerves enter to the retina of human eyes. An accurate identification of the optic disc boundary and changes in optic disc shape and area may be can be used to indicate the some diseases progression, especially glaucoma [2][3][4][5].

There are more than 60 million glaucoma cases in the world, and by 2020, the cases are predicted to increase to 80 million, particularly for adults with age of more than 40 years. Glaucoma is the second leading cause of blindness after refractive and cataract. There are more than 60 million glaucoma cases in the world, and by 2020 , the cases are predicted to increase to 80 million, particularly for adults with age of more than 40 years [6][7].

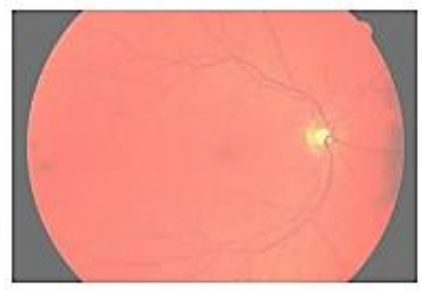

(a)

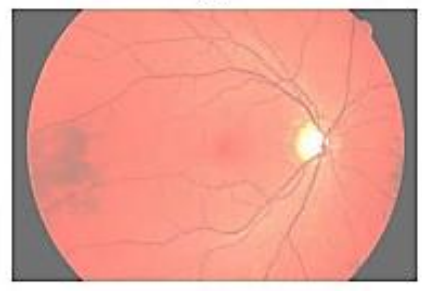

(b)

Fig. 1. Example of (a) glaucoma and (b) healthy images of High Resolution Fundus (HRF) image database [8] 
Diabetes is one of the diseases that occurs when the pancreas organ does not secrete enough insulin or the body is unable to process the insulin secreted by pancreas properly. It results in an abnormal condition where the glucose level in the blood is increased. Moreover, this high level of glucose causes damage to blood vessels in pancreas and others. Meanwhile, around 387 million world wide's people in 2014 lived with diabetes. Unfortunately, there are about $46.3 \%$ people undiagnosed to be diabetes addicted. Every seven seconds, a person dies 3 because of diabetes in 2014 according to International Diabetes Federation (IDF) [9][10][11].

Several studies on how optic disc centre is detected have been conducted. Kaur et al. [12] proposed optic disc boundary detection technique. In this method, blood vessel was eliminated with morphological closing technique, and optic disc boundary was segmented using active contour model (ACM). Another approach has been proposed by Akram et al. [13]. This work was based on canny edge detection method and Hough transform in order to detect the optic disc boundary. The proposed method was tested on DRIVE, STARE, diaretdb0 and diaretdb1 databases of manually labelled images. Further work on optic disc and cup segmentation for glaucoma assessment has been conducted by Joshi et al. [14]. Optic cup segmentation method is developed which is based on anatomical evidence such as vessel bends at the cup boundary. The method has been validated on 138 images consist of 33 normal and 105 glaucomatous images against three glaucoma experts.

Based on aforementioned backgrounds, optic disc centre automatic detection is a popular issue. In this research, an automated method of optic disc centre detection is proposed and employed in healthy and unhealthy retinal colour images. Optic disc centre as the results of the proposed method, can be used as an alternative to identify retinal related diseases especially glaucoma and diabetic retinopathy.

\section{Theoretical Basis}

\section{II.1. Eye}

The eye is a special sense organ made up of three coats. The outer fibrous layer of connective tissue forms the cornea and sclera. The middle vascular layer is composed of the iris, ciliary body, and choroid. The inner neural layer is the retina. To provide binocular vision, the muscles of both eyes are coordinated. The neural signal that carries visual information passes through a complex and intricately designed pathway within the central nervous system, enabling an accurate view of the surrounding environment. This information, evaluated by a process called visual perception, influences myriad decisions and activities [15].

The optic disc or optic nerve head is the part of the optic nerve which can be seen on assessment of eye. It is composed of $1,200,000$ tiny nerve fibres that send signals from the eye to the brain. The typical optic disc is a circular structure where the nerve fibres exit the eye and on average, only 1.5 $\mathrm{mm}$ in diameter. The optic disc begins in the eye. In the optic disc, there exists nerve fibres emerge. Each nerve fibres receives visual signals from a certain area of retina. This signal represents an area of one's field of vision in retina. The disc area is larger than the area taken up by the nerve fibres leaving the eye, so a small area of the central optic disc is left "unfilled" forming a small depression, called the optic cup [17].

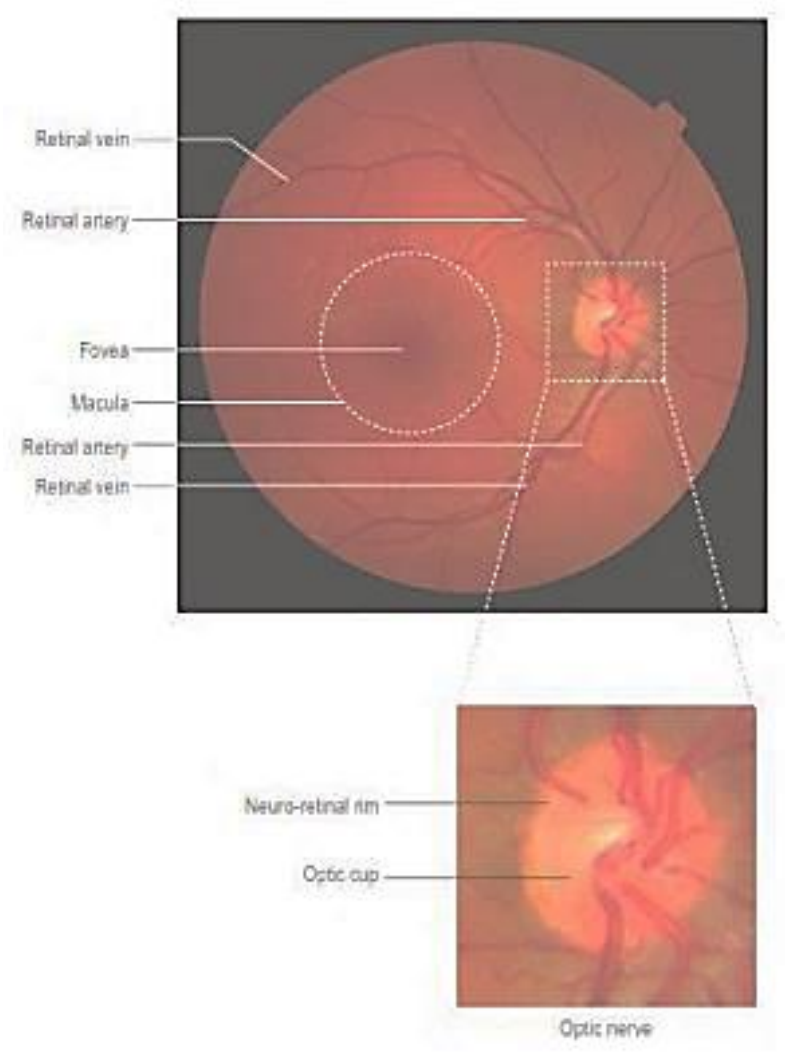

Fig. 2. The anatomy of eye in retinal image [16]

\section{II.2. Image Processing}

The digital colour retinal images are required to develop automatic system of retinal related diseases 
detection. The images are captured by fundus camera and consists of 8-bits of Red, Green and Blue RGB) layers with 256 levels each. However, patient movement, poor focus, bad positioning, reflections, inadequate illumination are some factors that can result a poor images of quality and cause the analysis becomes more difficultly [18]. To overcome this, several image processing techniques on such images can improve the quality of images and level of success in the automated retinal related disease detection [19].

\section{Proposed Study}

The aim of this chapter is to describe the proposed method for automated detection of optic disc centre in retina fundus image to diagnose of retinal related diseases. This chapter begins by presenting the data used in this research work. Afterwards, the tools of this research work describes the research flow and the image processing technique overview. The flow of the study in this paper can be visualized in a flow diagram as shown in Figure 1.

To perform detection of optic disc, especially in diameter of optic disc, High Resolution Fundus (HRF) database was used [8]. This database consists of 15 images of healthy patients, 15 images of patients with diabetic retinopathy and 15 images of glaucomatous patients. The images were captured using Canon CR-1 fundus camera a field of view of $45^{\circ}$ and different acquisition setting. The first step of this method is to detect the optic disc centre point candidate. All steps conducted to detect the optic disc centre point in this research work are depicted in Figure 3.

The retinal fundus image whether from Messidor or HRF database consists of three components, namely the Red, Green and Blue (RGB) Channels. The RGB (Red, Green, and Blue) colour space is one of the most used colour spaces, particularly for 8 bit digital images as expressed in (2-1) [8][20][21]. Red channel is the brightest image, the green channel has the best contrast and the blue channel image has the worse brightness and contrast [6]. Thus, green channel is extracted. Then, optic disc is localised using average filter. Average filter can be performed using equation (2-2). This filter is implemented by creating square average mask which has $51 \times 51$ of size. After that, the coordinates of some pixels having highest intensity value in retinal image are determined and then their centre point coordinate is obtained to produce the optic disc centre point candidate. By measuring the median coordinate value of row and column of all of the maximal pixels value, optic disc point candidate is determined.

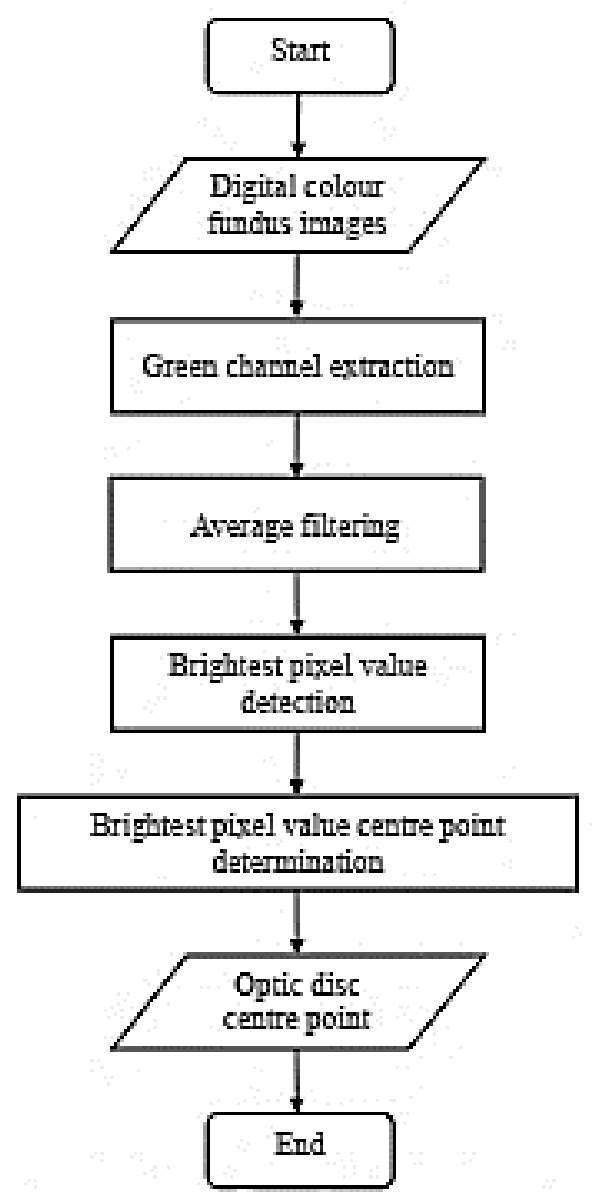

Fig. 3. Optic disc point candidate method

\section{Result}

Detected optic disc centre point candidate is used in two parts of this proposed research. In this chapter, the description of the used of optic disc centre point candidate is focused on its application as a feature of glaucoma diseases. The example of HRF original colour image consists of red, green, and blue channel can be seen in Figure 4 .

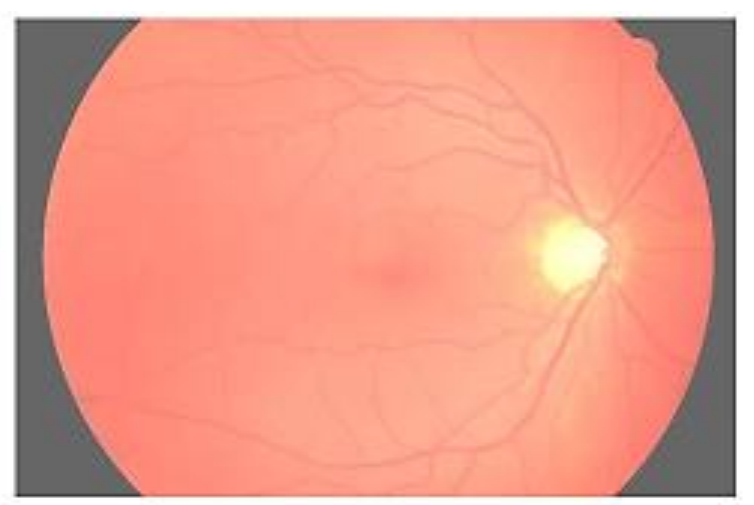

Fig. 4. Sample of HRF dataset [4] 
The proposed method is started by choosing the green channel due to having the highest contrast compared to the others as shown in Figure 5.

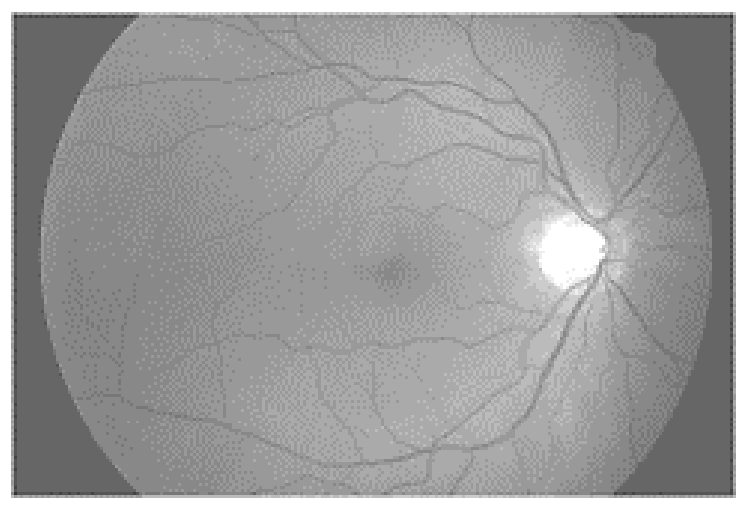

Fig. 5. Green channel extraction

Filtering is applied in the next step. Average filtering providing blurred effect in image is worked in $51 \times 51$ of size of window. Then, the next step is done by extracting the pixel coordinate having brightest or highest intensity value. Finally, the optic disc centre point candidate is achieved by determining the centre of the extracted pixel coordinate in the previous step. An example of detected optic disc centre point can be seen in Figure 6 below which is marked with the blue marker.

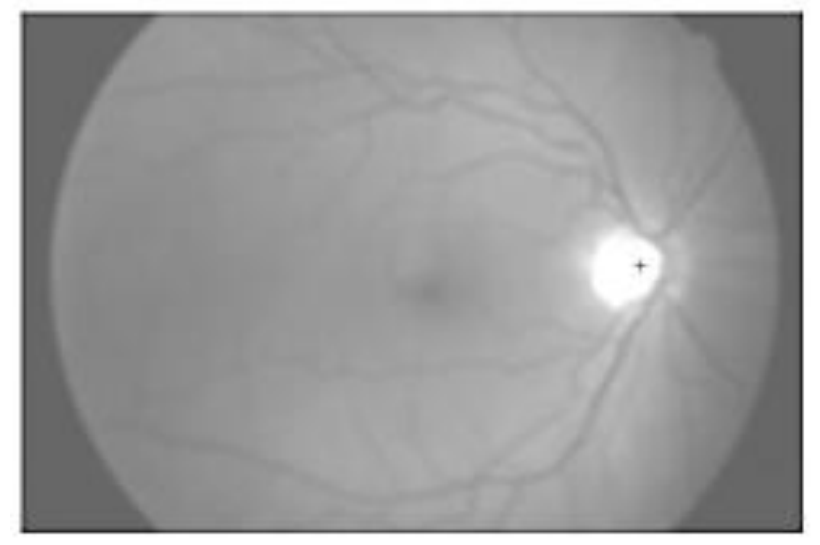

Fig. 6. Optic disc centre point candidate in retinal image

\section{Conclusion}

The implementation of detection of optic disc centre retinal colour fundus images to facilitate the diagnosis of retinal related diseases has been conducted. This research work proposes an approach to assist ophthalmologists in making decision in terms of diagnosis retinal related diseases based on automatically of optic disc centre detection. Some of previous works have their own limitations, particularly in automation view and only work in healthy retinal image. Therefore, there is not information on how optic disc centre in retinal image of unhealthy patient can be extracted computationally.

\section{References}

[1] Z. Zhang, H. Lee, J. Liu, W. K. Wong, N. M. Tan, and J. Hwee, "Optic Disc Region of Interest Localization in Fundus Image for Glaucoma Detection in ARGALI," Conference on Industrial Electronics and Applicationsis, pp. 1686-1689, 2010

[2] M. K. Dutta and A. K. Mourya, "Glaucoma Detection by Segmenting the Super Pixels from Fundus Colour Retinal Images," International Conference on Medical Imaging, m-Health and Emerging Communication Systems (MedCom), pp. 86-90, 2014.

[3] A. P. Shibal Bhartiya, Ritu Gadia, Harinder S Sethi, "Clinical Evaluation of Optic Nerve Head in Glaucoma," Clinical Evaluation of Optic Nerve Head in Glaucoma, vol. vol. 92, pp. 115-132, 2010

[4] R. Odstrcilik, Jan. Kolar, "Analysis of Retinal Image Data To Support Glaucoma Diagnosis," ÚSTAV BIOMEDICÍNSKÉHO INŽENÝRSTVÍ, 2014.

[5] P. K. Kohli, "Exact Detection of Optic Disc in Retinal Images using Segemntation based on Level Set Method and Morphology Operations," Thapar University, Patiala, pp. 1-59, 2012.

[6] H. A. Nugroho, L. Listyalina, N. A. Setiawan, S. Wibirama, and D. A. Dharmawan, "Automated Segmentation of Optic Disc Area using Mathematical Morphology and Active Contour," International conference on computer, control, informatics, and its applications, pp. 18-22, 2015.

[7] M. Zubair, "Automated Detection of Optic Disc for the Analysis of Retina Using Color Fundus Image," IEEE Int. Conf. Imaging Syst. Tech, pp. 239 - 242, 2013.

[8] P. Choukikar, A. K. Patel, and R. S. Mishra, "Segmenting the Optic Disc in Retinal Images Using Bi- Histogram Equalization and Thresholding the Connected Regions," International Journal of Emerging Technology and Advanced Engineering, vol. 4, no. 6, 2014.

[9] V. M. Mane and D. V. Jadhav, "Review: Progress Towards Automated Early Stage Detection of Diabetic Retinopathy: Image Analysis Systems and Potential," Journal of Medical and Biological Engineering, vol. 34, 2014.

[10] I. D. Federation, "Diabetes : Facts and Figures," Diabetes, p. 3, 2014.

[11] P. Z. Alberti, K. G., and Zimmet, "Definition, diagnosis and classification of diabetes mellitus and its complications, part 1: diagnosis and classification of diabetes mellitus provisional report 
of a WHO consultation," Diabet. Med., pp. 15(7):539-553, 1998.

[12] J. S. Kaur, "Automated Localisation of Optic Disc and Macula from Fundus Images," International Journal of Advanced Research in Computer Science and Software Engineering, vol. 2, no. 4, pp. 242249, 2012.

[13] M. U. Akram, A. Khan, K. Iqbal, and W. H. Butt, "Retinal Images: Optic Disk Localization," International Conference on Image Analysis and Recognition, pp. 40-49, 2010.

[14] G. D. Joshi, J. Sivaswamy, and S. R. Krishnadas, "Optic Disk and Cup Segmentation From Monocular Color Retinal Images for Glaucoma Assessment," IEEE Transactions on Medical Imaging, vol. 30, no. 6, pp. 1192-1205, 2011.

[15] Lee Ann Ramington, Clinical Anatomy and Physiology of The Visual System, no. 1. 2014.

[16] P. Gosh, Collier. Varikarra, "Fundoscopy Made Easy: The Normal Fundus and Its Variants," Churchill Livingstone, Elsevier, p. 21, 2010.

[17] Glaucoma NZ to safe sight, "Your Glaucoma Eye Examination: Part 2 Your Optic Disc," Glaucoma, vol. 5, no. 2, pp. 8-9, 2015.

[18] M. P. Waghmare, S. D. Chede, and P. S. M. Sakhare, "Design Strategies for Classification of Abnormalities in Retinal Images Using ANFIS," International Journal of Application or Innovation in Engineering \& Management, vol. 3, no. 3, pp. 388393, 2014.

[19] M. Sahebrao, Raju. N, Sangramsing. Meldhe, Sandip T. Dhopeshwarkar, “Automated Diagnosis Non-proliferative Diabetic Retinopathy in Fundus Images using Support Vector Machine," International Journal of Computer Applications, vol. 125, no. 15, pp. 7-10, 2015.

[20] K. J. Zana F, "A Multi-Modal Registration Algorithm of Eye Fundus Images Using Vessels Detection and Hough Transform," IEEE transactions on medical engineering, vol. vol. 18 no, pp. 419-428, 1999.

[21] Automated localisation of optic disc in retinal colour fundus image for assisting in the diagnosis of glaucoma. L Listyalina, HA Nugroho, S Wibirama, WKZ Oktoeberza. Communications in Science and Technology 2 (1)

\section{Authors' information}

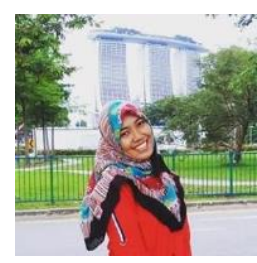

Latifah Listyalina is a lecturer at the Department of Electrical Engineering, Faculty of Science and Technology, Universitas Respati Yogyakarta. She received the B.Eng. degree in Biomedical Engineering from Universitas Airlangga, Indonesia, in 2013 and the M.Eng. degree in Electrical Engineering from Universitas Gadjah Mada, Indonesia in 2016. Her research interests include biomedical signal and image processing, computer vision and pattern recognition.

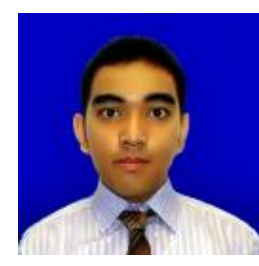

Dhimas Arief Dharmawan is a lecturer at the Department of Electrical Engineering, Faculty of Engineering, Universitas Muhammadiyah Yogyakarta. $\mathrm{He}$ received the B.Eng. degree in Electrical Engineering from Universitas Gadjah Mada, Yogyakarta, Indonesia in 2014. He is currently pursuing the Ph.D. degree in Electrical Engineering at Nanyang Technological University (NTU), Singapore. His research interests include image filtering and segmentation, machine learning, computer vision and pattern recognition. 